\title{
ANOMALOUS ORIGIN OF THE LETT CORONARY ARTERY FROM THE PULMONARY ARTERY WITH INTRAMURAL AORTIC ROUTE: DIAGNOSIS AND SURGICAL TREATMENT
}

\author{
Miguel Barbero-Marcial, MD, Carla Tanamati, MD, Edmar Atik, MD, Munir Ebaid, MD, and Adib Jatene, MD, \\ São Paulo, Brazil
}

Between 1987 and 1997, four patients with the diagnosis of an anomalous origin of the left coronary artery from the pulmonary artery (ALCAPA) were operated on at the Heart Institute and Hospital Sírio Libanês. Ages ranged from 3 to 15 months (mean age 8.5 months). During the operation, we observed that the left main coronary artery had an anomalous origin high on the right pulmonary artery or at the bifurcation of the main and right pulmonary arteries, passed behind the pulmonary trunk, entered the aortic wall, running intraluminally, and then was distributed normally to the left ventricle.

The patients included 3 boys and 1 girl. All patients had clinical evidence of congestive heart failure at the time of the initial evaluation, and all but 1 had an angina-like syndrome. Chest x-ray films showed cardiomegaly and electrocardiograms displayed evidence of myocardial infarction or ischemia. Preoperative echocardiography showed impaired left ventricular contraction. The ALCAPA was demonstrated angiographically in all cases, but in none was the intra-aortic route identified.

Operative technique. The operation was performed with cardiopulmonary bypass at $20^{\circ} \mathrm{C}$ to $25^{\circ} \mathrm{C}$. The aorta was crossclamped and cold blood cardioplegic solution was administered antegradely. The pulmonary arteries were occluded to maintain high pressure within the proximal pulmonary trunk.

The first patient was operated on in $1987 .^{1,2}$ After infusion of cardioplegic solution, the pulmonary trunk was opened without identification of the left coronary ostium. The dissection of the left coronary artery showed an anomalous origin, high on the right pulmonary artery. This coronary artery passed behind the pulmonary trunk, entered the aortic wall running parallel to the aorta, and then distributed normally to the left ventricle (Fig 1, A). One probe was introduced into the left coronary ostium, disclosing a suspicion of an intramural aortic route. The ascending aorta was opened wide and the vertical intramural trajectory was identified. The intra-

From the Departments of Cardiothoracic Surgery, Cardiology, Pediatric Cardiology, and Cardiac Surgery, Heart InstituteUniversity of São Paulo Medical School and Hospital, Sírio Libanês, São Paulo, Brazil.

Received for publication Aug 17, 1998; accepted for publication Nov 2, 1998.

Address for reprints: Miguel Barbero-Marcial, MD, Heart InstituteUniversity of São Paulo Medical School, Avenida Dr, Enéas de Carvalho Aguiar, 44, CEP 05403-000, São Paulo, Brazil.

J Thorac Cardiovasc Surg 1999;117:823-5

Copyright (C) 1999 by Mosby, Inc.

$0022-5223 / 99 \$ 8.00+0 \quad \mathbf{1 2 / 5 4 / 9 5 5 8 8}$ mural portion of the coronary artery was unroofed intraluminally and its intima was attached to the aortic intima with 12 interrupted 7-0 polypropylene sutures to avoid dissection of the aortic wall and occlusion of the aortocoronary window (Fig 1, B). The origin of the ALCAPA on the right pulmonary artery was then ligated and the aorta and pulmonary trunk were closed directly.

In 2 subsequent patients, the same procedure was used.

In 1 patient, direct implantation of the left coronary artery into the aorta was performed after dissection of the partially common coronary arterial wall and aortic wall. The main pulmonary artery was reconstructed with a patch of bovine pericardium.

Results. In the unroofed group no deaths were recorded over a follow-up period of 9 months to 10 years. All patients had an uneventful postoperative recovery and are clinically well, in functional class I (New York Heart Association) without any medication. The follow-up echocardiograms have shown improvement in myocardial function with mild mitral regurgitation in 2 of the patients. The attempt to dissect the partially common coronary arterial wall and aortic wall resulted in severe postoperative complications. This patient was progressing in New York Heart Association functional class II and suddenly died 6 months after the operation.

Discussion. The sites of coronary artery origin from the pulmonary trunk are variable. In cases of anomalous origin at the bifurcation of the main and right pulmonary arteries or from the right pulmonary artery, this coronary artery frequently follows a vertical route, behind the ascending aorta, changes its direction close to the pulmonary anulus, and then distributes normally to the left ventricle. In certain cases, the descending trajectory of this coronary artery takes place in the medial layer of the aorta.

The echocardiogram, in our experience, failed to detect the aortic intramural course. This failure can be explained by the intimal relationship between the aorta and anomalous left coronary artery, by the absence of visualization of the anomalous origin into the pulmonary trunk, and by its vertical course, parallel to the ascending aortic wall. Sanders (personal communication, 1998) suspected that this failure was due to dropout of the thin endocardial wall between the coronary artery and the aorta. Cardiac catheterization is the most sensitive method for a definitive diagnosis. The preoperative selective injection of contrast media into the right coronary artery identified the ALCAPA but not the intramural aortic course in our cases. Retrospectively, careful analysis of the angiograms showed some indications that suggested the pres- 


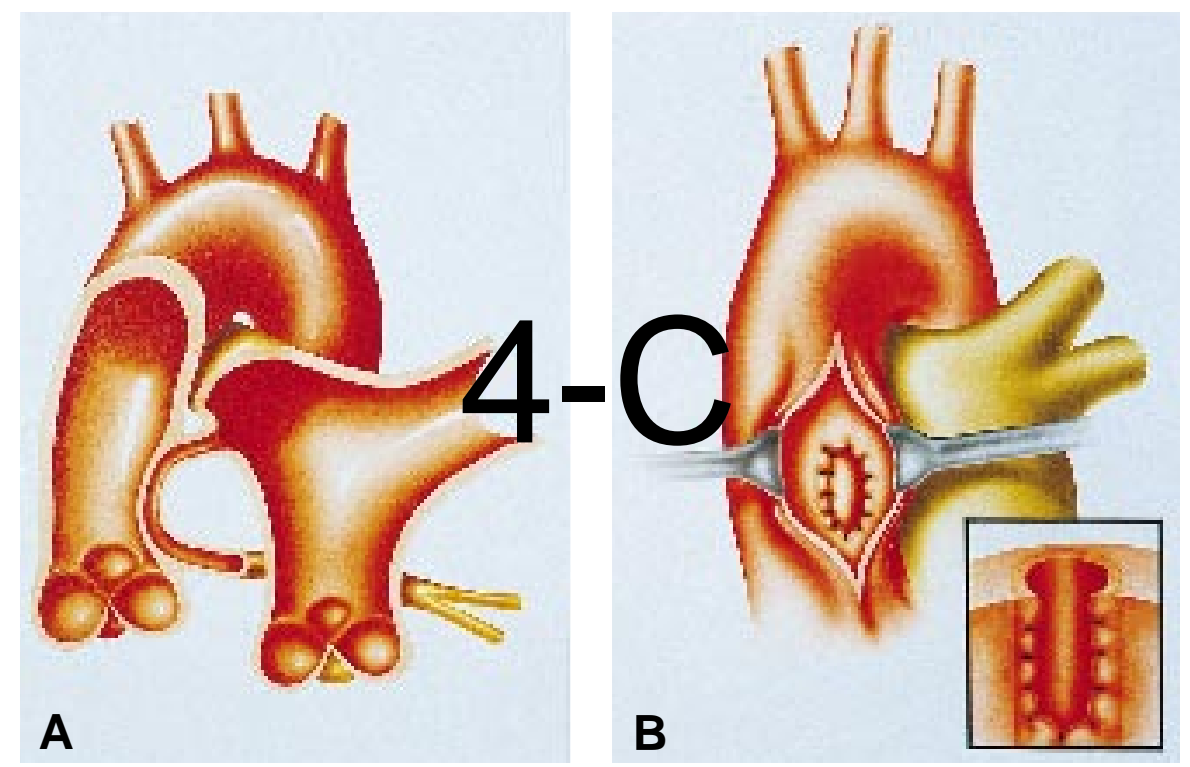

Fig 1. Surgical illustration of the aortocoronary window. A, Relationship between the anomalous left coronary artery and the aortic wall. The left coronary artery had an anomalous origin, high on the right pulmonary artery, passed behind the pulmonary trunk, entered the aortic wall running parallel to the aorta, and then was distributed normally to the left ventricle. $\mathbf{B}$, The anterior wall of the ascending aorta was opened wide and the intramural portion of the coronary artery was unroofed intraluminally and its intima attached to the aortic intima with 12 interrupted sutures. Inset shows the final appearance of the created aortocoronary window.

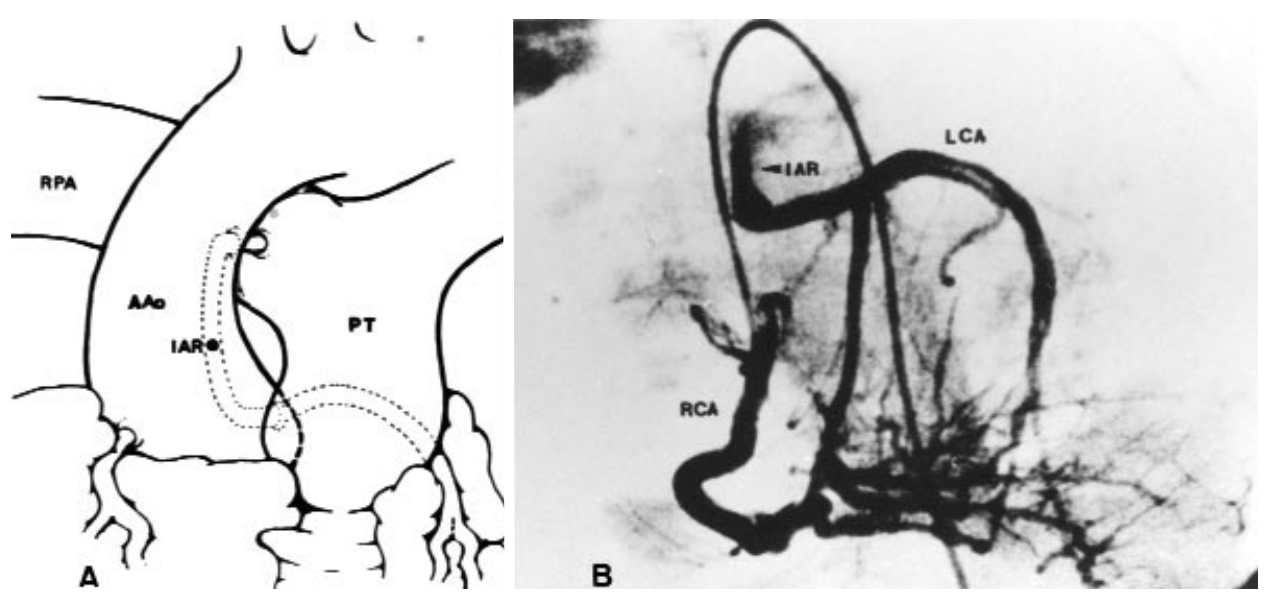

Fig 2. Cineangiogram in 1 patient with ALCAPA with an intramural aortic route. A, Trajectory of the anomalous left coronary artery and its relationship with the aorta and pulmonary trunk. B, Right anterior oblique projection showing some indications suggesting the presence of the intramural aortic route. RPA, Right pulmonary artery; $P T$, pulmonary trunk; $R C A$, right coronary artery; $L C A$, left coronary artery; IAR, intramural aortic route; $A A o$, ascending aorta.

ence of the intramural aortic route (Fig 2): (1) high origin of the left coronary artery at the pulmonary trunk, near the right pulmonary artery, or on the right pulmonary artery itself; (2) ascending and parallel course of the ALCAPA to the aortic wall; (3) presence of a left perpendicular angle formed by the junction of the vertical intramural aortic course and its subsequently horizontal portion until it reaches the left ventricle; and (4) superoinferior relationship between the anomalous ostium of the ALCAPA on the right pulmonary artery and the ostium of the right coronary artery. 
The ideal surgical treatment of ALCAPA consists in establishing 2 coronary artery systems.

In patients with a short intramural trajectory or in those in whom the anomalous coronary artery does not enter completely into the thickness of the aortic wall, separation of the 2 structures is difficult. However, this technique can be performed, as in 1 of our patients, if the wall of the anomalous coronary artery is protected.

Identification of the intramural aortic course, unroofing the coronary artery intraluminally, and attaching its intima to the aortic intima was described by our group in $1988^{1}$ and by Turley and colleagues ${ }^{3}$ in 1995 . The presented technique permits a simple and safe approach to this anomaly, creating a wide intraluminal aortocoronary window. The medium-term clinical results have been encouraging, in that all patients survived the procedure with improvement of functional class and of left ventricular function.
We thank Dr Stephen P. Sanders for his important contribution to this article.

\section{REFERENCES}

1. Atik E, Barbero-Marcial M, Ikari NM, et al. Anomalia isolada da artéria coronária esquerda. Trajeto inusitado dentro de parede da aorta ascendente e inseção na artéria pulmonar direita. Relato de caso. Arq Bras Cardiol 1988;51:335-9.

2. Atik E, Barbero-Marcial M, Ikari NM, Kajita L, Machado D, Ebaid $\mathrm{M}$, et al. Origem da artéria coronária esquerda das artérias pulmonares direita e esquerda-Avaliação clínica, anatomocirúrgica e evolutiva de três caso. Arq Bras Cardiol 1991;57: 121-7.

3. Turley K, Szarnicki RJ, Flachsbart KD, Richter RC, Popper RW, Tarnoff H. Aortic implantation is possible in all cases of anomalous origin of the left coronary artery from the pulmonary artery. Ann Thorac Surg 1995;60:84-9. 